\title{
Research on Laser Measurement System for Underground Unmanned Load-Haul-Dump
}

\author{
Li Jianguo ${ }^{*}, 1,2$, Zhan $\mathrm{kai}^{2}$, Li Hengtong ${ }^{2}$ and Guo Xin ${ }^{2}$ \\ ${ }^{I}$ School of Mechanical Engineering, University of Science and Technology, Beijing, 100083, P.R. China \\ ${ }^{2}$ Mechanical Department, Beijing General Research Institute of Mining and Metallurgy, Beijing, 100160, P.R. China
}

\begin{abstract}
A method of data processing for the laser measurement system (LMS) based on the software LABVIEW which is used for underground unmanned Load-Haul-Dump (LHD) is described. The background of the technology is recommended firstly, the characteristics of LABVIEW and LMS are introduced briefly. How to process the data from LMS using LABVIEW including the algorithm for decoding the data and the frame of programming thought is described in detail. The design of data processing system with a serial port debugging system is expounded. Finally, the system is applied on a model LHD and it is proved that the system has feasibility and wide applying prospects for unmanned LHD.
\end{abstract}

Keywords: LABVIEW, laser measurement system, LHD, serial.

\section{INTRODUCTION}

With the development of economy, the demand for mineral resources obtained by means of deep mining continues to increase rapidly. In the deep mining, the demand for the use of automatic mining equipment has also increased. For example, the repetitive "load-haul-dump" cycle is well suited to automation. In this case, a vehicle called a load-haul-dump (LHD) machine is often used to excavate fragmented rock, haul it to an assigned location, and then dump the material before returning for another load [1]. A number of reasons have led to the desire to automate the operation of LHD vehicles. First, the mine is generally not offering the best environment conditions for humans. Second, the nature of this task is such that the vehicle and its operator are continuously subject to the risk of being hit or buried by falling rocks, since the load operation is performed in unsecured areas. Third, an automated LHD vehicle could allow reduced operation costs and increased productivity. Fourth, automatic control of the LHD vehicle could lead to less mechanical strain, which would in turn reduce the maintenance costs [2].

For the automatic LHD, the key factor of controlling is how to identify the surrounding tunnel information which can used to guide the next action. As the established technologies, laser measurement system (LMS) and image are the two methods to apply for the controlling, compared with the image method, LMS method has many advantages, such as low cost, convenient operation etc. This article describes a method to process the data of LMS based on the software LABVIEW, and the data is used for a model LHD which can be driven automatically under the simulate tunnel.

\section{LASER MEASUREMENT SYSTEM}

The laser measurement system is a non-contact, standalone remote measuring system designed for use in industrial environments. It is usually used for distance measuring and obstacles detecting. LMS has been in extensive use for many years for autonomous vehicles or robots. Their primary usages are obstacle detection and as aid in localization.

\subsection{Operating Principle for LMS}

A laser measurement system is a device that uses a series of laser rays to measure the ranges to objects. In the underground tunnel, it is installed on the LHD and measures the ranges to the tunnel wall. As an example, one of the kinds of LMS which is applied in this article is shown in Fig. (1).

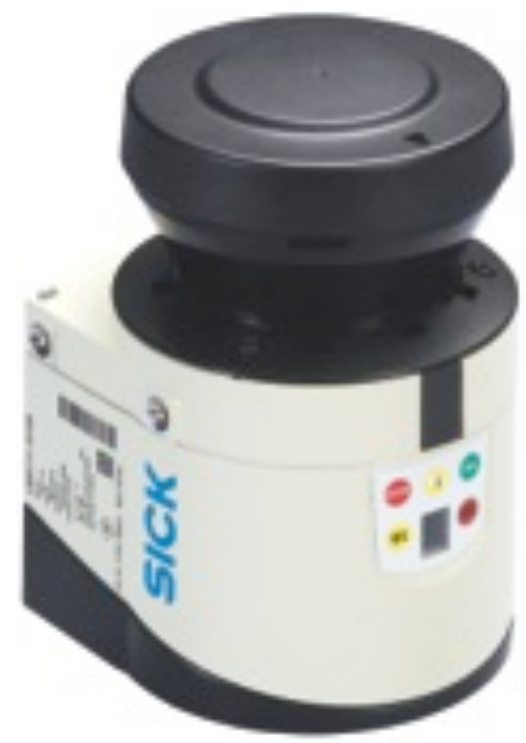

Fig. (1). Example of LMS. 
It has been chosen for the following reasons which are suitable for the automatic LHD:

1) The unit is specified for underground use, is waterproof, and can be equipped with heating equipment for very low temperatures.

2) The unit has fog correction for underground use which can improve the accuracy of data.

3) The wavelength is $902 \mathrm{~nm}$ which can also work underground.

4) The unit is operable over two different scanning angles $180^{\circ}$ and $100^{\circ}$ with $0.5^{\circ}$ angular resolution. These can improve the precision of the tunnel information.

The LMS operates by measuring the time of flight of laser light pulses: a pulsed laser beam is emitted and reflected if it meets an object as Fig. (2). The reflection is registered by the LMS's receiver. The time between transmission and reception of the impulse is directly proportional to the distance between the LMS and the object (time of flight) [3].
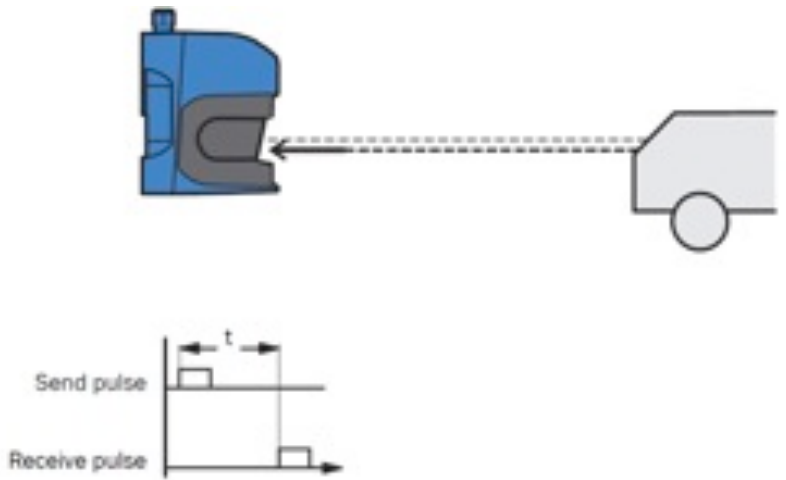

Fig. (2). Operating principle.

The pulsed laser beam is deflected by an internal rotating mirror so that a fan-shaped scan is made of the surrounding area. The contour of the target objects is determined from the sequence of impulses received. The measurement data is available in real-time for further evaluation via the data interface.

The range of angle measured can be chosen $180^{\circ}$ or $100^{\circ}$ and it supports a configurable angular resolution in steps of $1^{\circ}, 0.5^{\circ}$ and $0.25^{\circ}$. It requires 13.32 millisecond for a standard rotation $\left(180^{\circ}\right.$ range and $1^{\circ}$ resolution), which corresponds to a measurement rate at $75 \mathrm{~Hz}$. If steps of $0.25^{\circ}$ or $0.5^{\circ}$ have been configured, 2 or 4 mirror rotations are required.

\subsection{Measurement and Data Output}

The LMS communicates with host system via a switchable RS232/422 interface. The data format for transmitting data is set as 1 start bit, 8 data bits and 1 stop bit. The data transmission rate can be changed by send command to the LMS and the velocity would be up to $500 \mathrm{~K}$ under RS422 mode. The LMS normally responds to a send telegram with a response telegram. The LMS receives commands as steams of bytes through the serial port. When transmitting data, it sends back streams of bytes corresponding to distance measurements at a given angle [4]. A typical stream of data from the LMS is shown as Table 1.

While the angle range is $180^{\circ}$ and the step resolution is $0.5^{\circ}$, the structure of valid measurement data is defined as Table 2.

Table 2. Format of measurement values.

\begin{tabular}{|c|ccc|}
\hline Data Numbers & Measurement Data & \multicolumn{2}{c|}{ Corresponding to Angle } \\
\hline \hline 361 & $0^{\circ} 0.5^{\circ} 1^{\circ} 1.5^{\circ}$ & $\ldots \ldots$ & $179.5^{\circ} 180^{\circ}$ \\
\hline
\end{tabular}

\section{PROGRAM OF LABVIEW}

Laboratory Virtual Instrumentation Engineering Workbench (LABVIEW) is a platform and development environment for a visual programming language form National Instruments [5]. The programming language used in LABVIEW, also referred as $G$, is a data flowing programming language. Execution is determined by the structure of a graphical block diagram (the LV source code) which the programmer connects different function-nodes by drawing wires. These wires propagate variables and any node can execute as soon as all its input data become available. Because of this might be the case for multiple nodes simultaneously, $\mathrm{G}$ is inherently capable of parallel execution. The data-flow (which can be forced, typically by linking input and outputs of nodes) completely defines the execution sequence, and that can be fully controlled by the programmer. Thus, the execution sequence of the LABVIEW graphical syntax is as well-defined as with any textually coded language such as $\mathrm{C}$, Visual Basic etc.

Table 1. Telegram structure.

\begin{tabular}{|c|c|c|c|}
\hline \multicolumn{2}{|c|}{ Description } & Data Length in Bytes/Data Class & Explanation \\
\hline \multicolumn{2}{|c|}{ STX } & 1/BYTE & Start byte \\
\hline \multicolumn{2}{|c|}{ Address } & 1/BYTE & Address of the subscriber \\
\hline \multicolumn{2}{|c|}{ Length } & 2/WORD & Number of subsequent data byte \\
\hline \multicolumn{2}{|c|}{ Command/response } & 1/BYTE & Command/response \\
\hline \multirow{2}{*}{ Data } & data & $2 * n$ & Measurement data \\
\hline & status & 1/BYTE & Status \\
\hline Checksum & & 2/WORD & CRC checksum \\
\hline
\end{tabular}


Another one important benefit of using LABVIEW over other programming language is the extensive support for accessing instrumentation hardware. Drivers and abstraction layers for many different types of instruments and buses that present themselves as graphical nodes are included or are available for inclusion. The abstraction layers offer standard software interfaces to communicate with hardware devices which can save program development time. For all these reasons, even people with limited coding experience can write programs and deploy test solutions in a reduced time frame when compared to more conventional or competing systems.

\subsection{Frame of Program}

Combination with the LMS operation, the structure of performance, data structure, communication protocol and the working principle of LMS, the program for data processing system is designed as the following operations shown in Fig. (3).

The LMS communicates with host system via a switchable RS232/422 interface. It is best to choose the virtual Instrument software architecture (VISA) model as the program assistant while considering the extensibility and opening of the software. The VISA is a standard for configuring, programming, and troubleshooting instrumentation systems comprising GPIB, VXI, PXI, Serial, Ethernet, and/or USB interfaces. This can help us to accelerate the work.

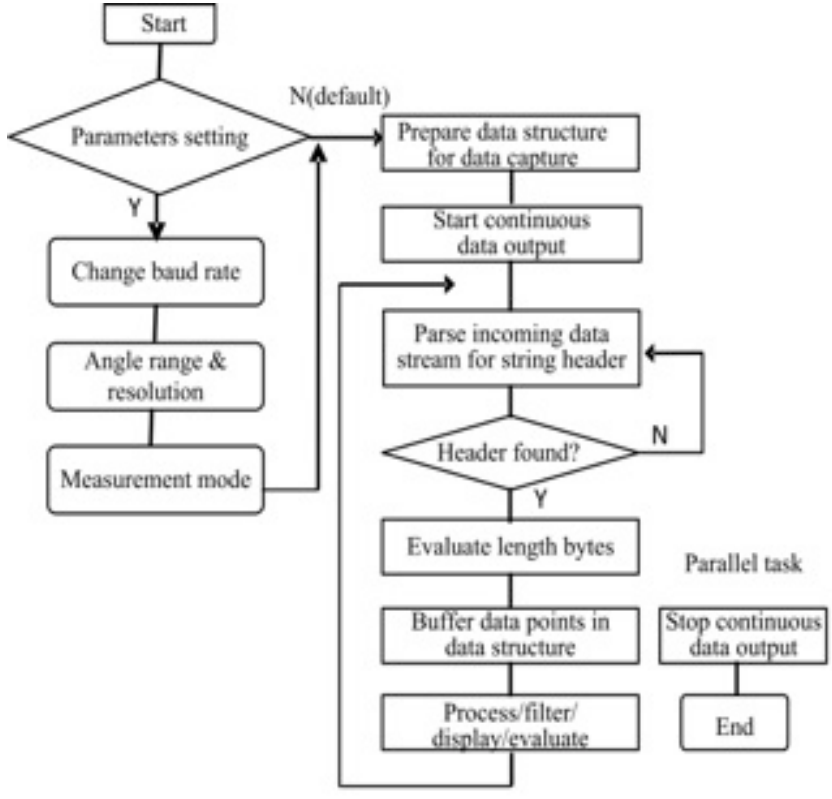

Fig. (3). Frame of program.

\subsection{Program}

LABVIEW programs/subroutines are called virtual instruments (VIS). Each VI has three components: a block diagram, a front panel, and a connector pane. The last is used to represent the VI in the block diagrams of other, calling VIS. However, the front panel can also serve as a programmatic interface. Thus a virtual instrument can either be run as a program, with the front panel serving as a user interface, or, when dropped as a node onto the block diagram, the front panel defines the inputs and outputs for the given node through the connector pane. This implies each VI can be easily tested before being embedded as a subroutine into a larger program. In the development of program, some controls and indicators are adopted. These controls and indicators on the front panel allow the operator to input command data into or extract data from the running virtual instrument.

A serial port debugging system is added in order to debugging the LMS conveniently [6]. The serial port debugging system is mainly composed of three parts: Ports setting model, data sending model and data receiving model. The port setting model can change the baud rate, start bit, data bit and stop bit etc. Data sending model allows operator to send data automatically or manually. Data receiving model allows operator to choose data display mode, such as decimal, hexadecimal or ASCII characters. It receives data automatically while there is data in the serial port. The block diagram of this debugging system is shown as Fig. (4).

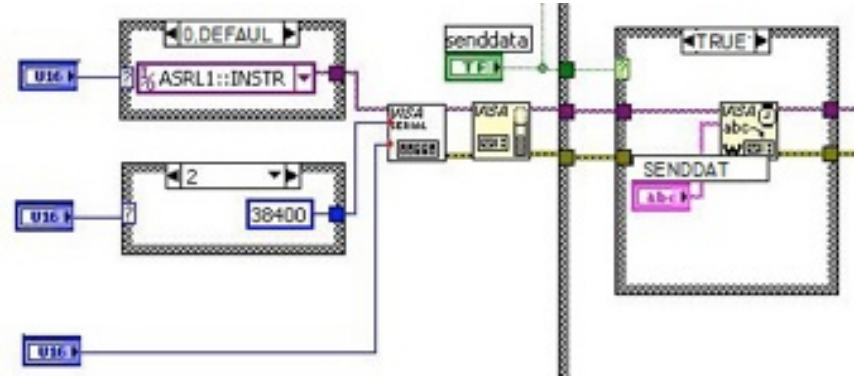

Fig. (4). Serial port debugging system.

In order to check the transmission data clearly, it is important to remember that it must send and receive BINARY data but not ASCII characters.

After a power-on of the LMS, the baud rate is reset to 9600 , the angle and resolution is reset to $180^{\circ} / 0.5^{\circ}$, the default measurement mode is reset to $\mathrm{cm}$ mode. In order to process the measurement data at a higher baud rate and change the measuring angle or distance, it is required to send command data to the LMS. All of these can be designed into a sub-Vi and the operator can choose one mode by pressing button on the front panel. The front panel is shown as Figs. $(5,6)$.

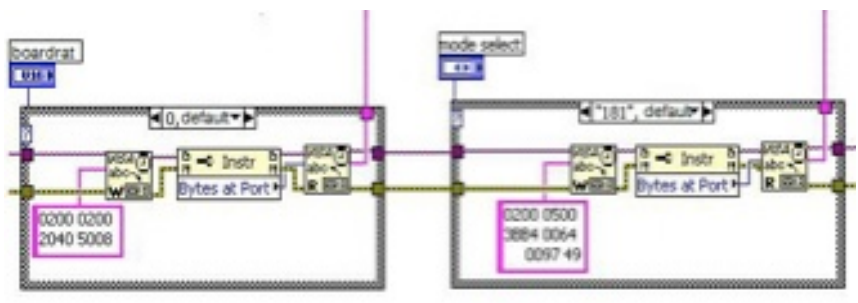

Fig. (5). Baud set and LMS mode.

It is noted that after each setting telegram, a reply telegram from the LMS need to be confirmed by the host. Only the complete reply telegram having received, the setting has been successfully implemented in the LMS and the next telegram can be sent [7]. It can take up to 7 seconds to receive the reply telegram from the LMS while changing 
between $\mathrm{cm}$ and $\mathrm{mm}$ mode, so the delay should be added into the program.

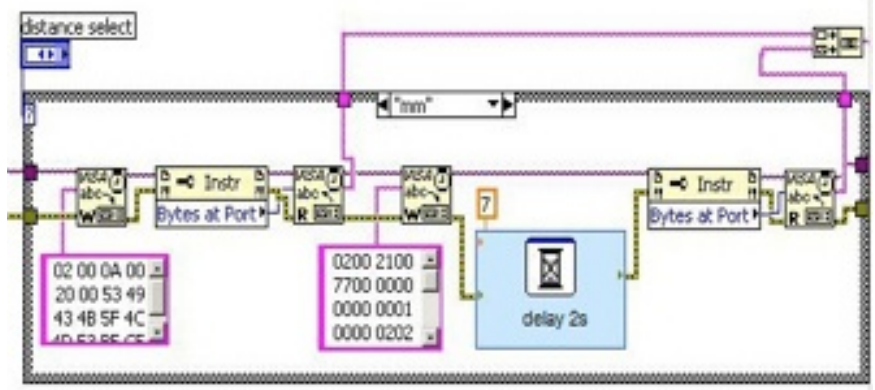

Fig. (6). Parameters setting of LMS.

Form section 2, the format of measurement data output values is known. In order to identify the start of an LMS output data string, it is necessary to acquire a specific header in the data stream. The output data string header is different from each measurement mode. Each measurement data value is composed of two bytes incessantly. It is necessary to strip the upper 3 bits of each value. The block diagram of identifying the header and stripping the upper 3 bits is shown in Fig. (7).

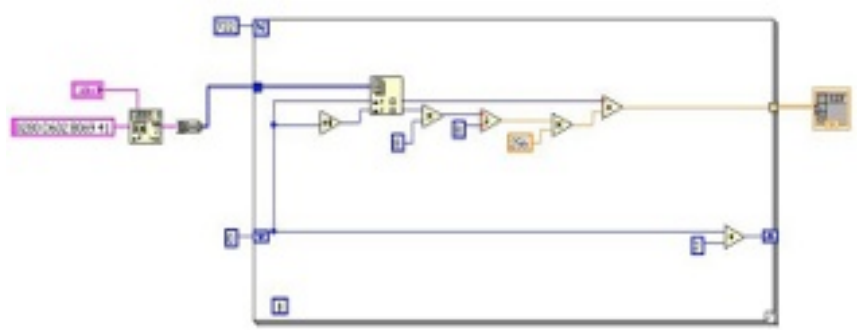

Fig. (7). Header indentify and data processing.

After the separate function models having completed, the whole block diagram of the data processing system is done.

\section{TEST AND APPLYING}

\subsection{LMS Testing}

A test is done to testify the program. In the test, we connect the LMS to PC and use the program to acquire the 2$\mathrm{D}$ environment front the LMS. The real time data is received and display in the front panel. The result is shown as Fig. (8).

\subsection{Applying on the Model LHD}

In studying the LMS and conducting experiments, we designed a scale model of the LHD shown as Fig. (9). The model LHD is an articulated four-wheel drive unit with steering function achieved through the steering cylinder. When the steering cylinder is elongated or shortened, the pre-and post-body articulated body angle become large or small [8]. The LMS is installed on the LHD model.

Reactive navigation is a widely controlling method for underground automatic LHD. In this mode, for the LHD to move through the environment, it does not need to "know" with any accuracy where it is in the environment with respect to some global co-ordinate frame. It only needs to know where it is relative to the navigation infrastructure or local objects. Reactive navigation systems do not need to perform any path planning at the global scale and may not even need path planning at the local scale [9]. Tunnels in underground mines are somewhat like corridors in an office building in that they have a floor, ceiling and walls.

The vehicle is controlled by an industrial computer (IPC), which issues steering and velocity commands, and sends encoder information that describes a trajectory. In this system, the IPC communicates with the internal A/D controller boards to control vehicle motion.

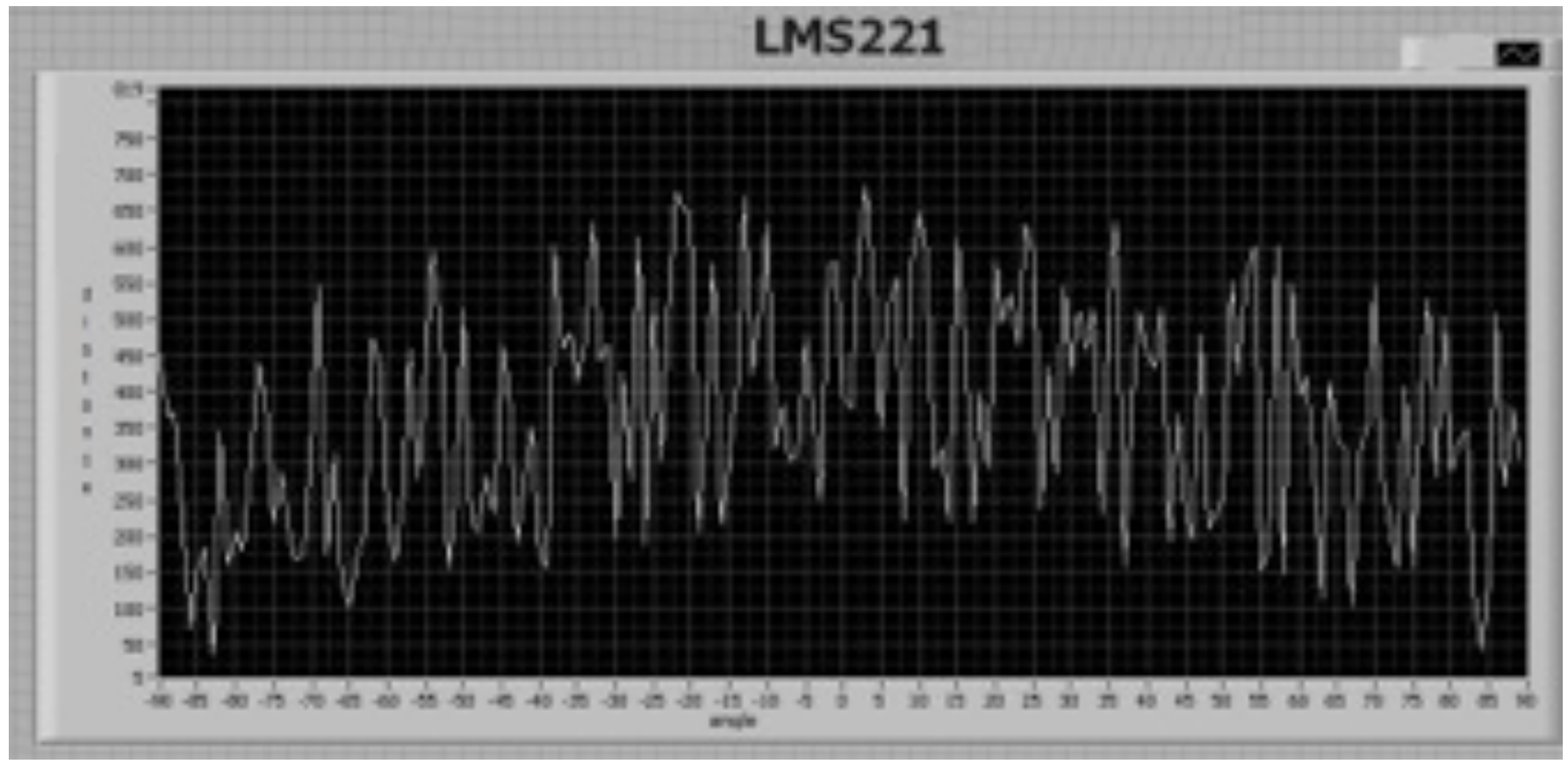

Fig. (8). Real-time measuring data processing of LMS. 


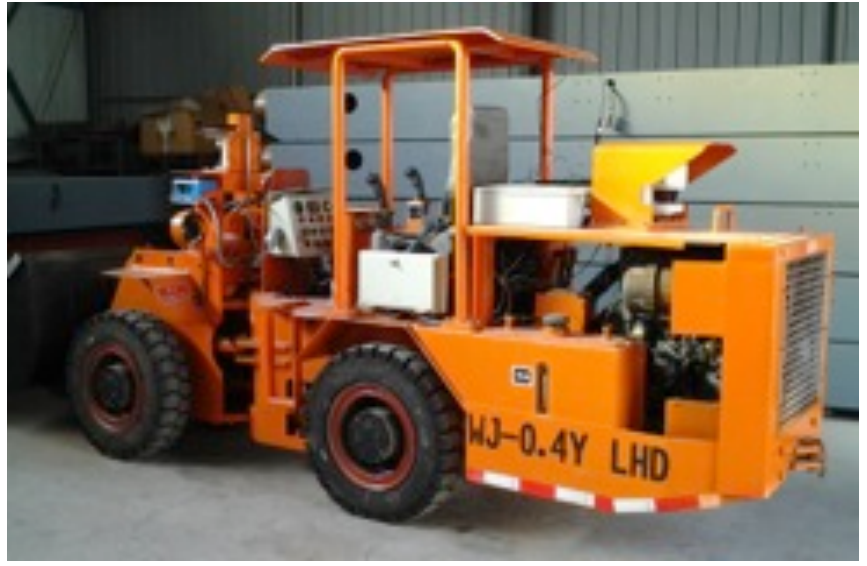

Fig. (9). LMS Work on LHD.

In this experiment, the aim of the model LHD controlling is driving under a given corridor environment shown as Fig.

\section{CONCLUSION}

We have developed LMS system suitable for underground unmanned LHD. The laser measurement systems are used as the primary sensors which are programmed using LABVIEW. Experiments show that the LMS can be used in navigation and unmanned control of model LHD, and the result proves that the system has feasibility and wide applying prospects for unmanned LHD.

\section{CONFLICT OF INTEREST}

The author confirms that this article content has no conflict of interest.

\section{ACKNOWLEDGEMENTS}

The authors are grateful to the financial supported by the National High Technology Research Program of China

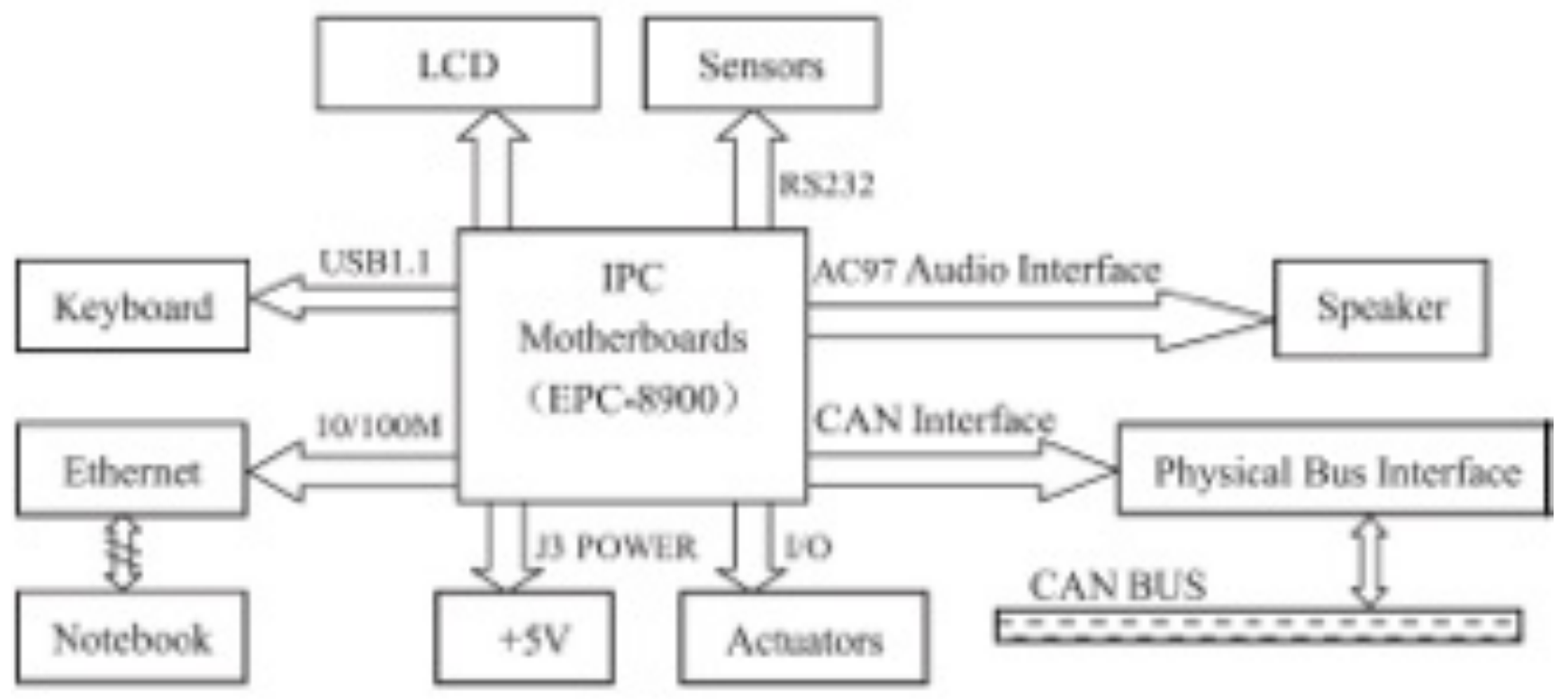

Fig. (10). IPC control function.

(11). During the driving, the LMS detects the front district which can use to control the velocity of the LHD and avoid the wall. The key factor of the automatic driving is keeping the distance from left equal to the right. The dashed line is the real trajectory of the LHD.

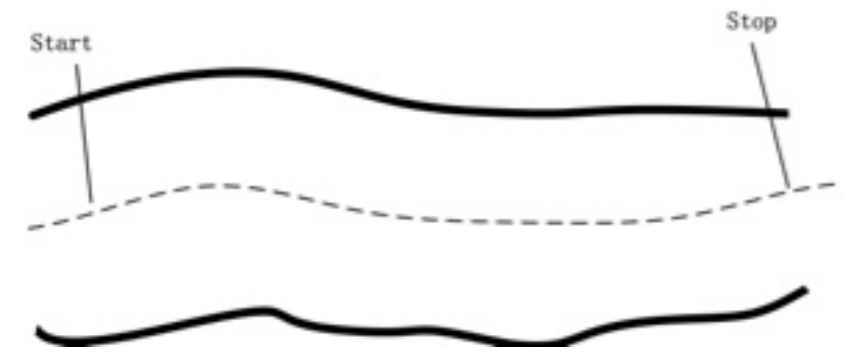

Fig. (11). Experiment map.
("863'" Project)(Grant No. 2011AA060403). The research also got support from the opening research funds from Beijing General Research Institute of Mining \&Metallurgy.

\section{REFERENCES}

[1] J.Larsson, M.Broxvall, and A.Saffiotti, "A navigation system for automated loaders in underground mines", in Field and Service Robotics, ser. Springer Tracts in Advanced Robotics, P. Corke and S. Sukkarieh, Eds. Berlin, DE: Springer, 2006, pp. 129-140, online at http://www.aass.oru.se/ asaffio/

[2] Joshua A. Marshall and Timothy D. Barfoot. Design and field testing of an autonomous underground tramming system. Springer Tracts in Advanced Robotics, 42:521-530, 2008.

[3] Arsalan Siddiqui, Thomas Hellström. Laser-based localization of vehicles and robots in natural and unstructured environments. 2005.

[4] Telegrams for Operating/Configuring the LMS 2xx Laser Measurement System (Firmware Version V2.10). SICK AG. Division Auto Ident, Germany. 
[5] http://en.wikipedia.org/wiki/LabVIEW

[6] J.Wang, J. Y. Zheng, "The design of serial port debugging system based on LabVIEW", Jiangxi Science, China, Dec, 2007, pp.756

[7] Quick Manual for LMS communication setup, Hardware setup and measurement mode configuration (Version 1.1), SICK AG. Division Auto Ident, Germany.
[8] Hongpeng Chi, Kai Zhan, Boqiang Shi, "Automatic guidance of underground mining vehicles using laser sensors", Tunnelling and Underground Space Technology 27(2012), pp.142-148.

[9] J. M. Roberts, E. S. Duff, P. I. Corke, P. Sikka, G. J. Winstanley, and J.B.Cunningham. Autonomous control of underground mining vehicles using reactive navigation. In Proceedings of the 2000 IEEE Conference on Robotics and Automation, pages 3790-3795, San Francisco, CA, April 2000

(C) Jianguo et al.; Licensee Bentham Open.

This is an open access article licensed under the terms of the (https://creativecommons.org/licenses/by/4.0/legalcode ), which permits unrestricted, non-commercial use, distribution and reproduction in any medium, provided the work is properly cited. 